\title{
Hyperpolarized 13C MR angiography
}

\section{Lipsø, Hans Kasper Wigh; Magnusson, Peter; Ardenkjær-Larsen, Jan Henrik}

\section{Published in:}

Current Pharmaceutical Design

Link to article, DOI:

$10.2174 / 1381612822666151109112415$

Publication date:

2016

Document Version

Peer reviewed version

Link back to DTU Orbit

Citation (APA):

Lipsø, H. K. W., Magnusson, P., \& Ardenkjær-Larsen, J. H. (2016). Hyperpolarized 13C MR angiography. Current Pharmaceutical Design, 22(1), 90-96. https://doi.org/10.2174/1381612822666151109112415

\section{General rights}

Copyright and moral rights for the publications made accessible in the public portal are retained by the authors and/or other copyright owners and it is a condition of accessing publications that users recognise and abide by the legal requirements associated with these rights.

- Users may download and print one copy of any publication from the public portal for the purpose of private study or research.

- You may not further distribute the material or use it for any profit-making activity or commercial gain

- You may freely distribute the URL identifying the publication in the public portal

If you believe that this document breaches copyright please contact us providing details, and we will remove access to the work immediately and investigate your claim 


\section{Hyperpolarized ${ }^{13} \mathrm{C}$ MR angiography}

Kasper W. Lips $\emptyset^{1,2}$, Peter Magnusson $\mathrm{PhD}^{2}$, and Jan Henrik Ardenkjær-Larsen $\mathrm{PhD}^{1,2,3}$

\section{Author affiliations:}

${ }^{1}$ Department of Electrical Engineering, Technical University of Denmark, Kgs. Lyngby, Denmark

${ }^{2}$ Danish Research Centre for Magnetic Resonance, Centre for Functional and Diagnostic Imaging and Research, Copenhagen University Hospital Hvidovre, Denmark

${ }^{3}$ GE Healthcare, Brøndby, Denmark

Corresponding author and Reprint info:

Jan Henrik Ardenkjær-Larsen, DTU Electrical Engineering, Ørsteds Plads, Building 349, room 106, 2800 Kgs. Lyngby, Denmark. Tel (0045) 4525 3918. E-mail: jhar@elektro.dtu.dk

\section{Keywords:}

Magnetic resonance angiography, hyperpolarization, ${ }^{13} \mathrm{C}, \mathrm{HP} 001$, DNP 


\begin{abstract}
Magnetic resonance angiography (MRA) is a non-invasive technology that can be used for diagnosis and monitoring of cardiovascular disease; the number one cause of mortality worldwide.

Hyperpolarized imaging agents provide signal enhancement of more than 10,000 times, which implies large reduction in acquisition time and improved spatial resolution. We review the role of hyperpolarized ${ }^{13} \mathrm{C}$ agents for MR angiography and present the literature in the field. Furthermore, we present a study of the benefit of intraarterial injection over intravenous injection of hyperpolarized agent for cerebral angiography in the rat, and compare the performance of two standard angiographic pulse sequences, the gradient echo (GRE) sequence and the balanced steady-state free precession (bSSFP).
\end{abstract}

2D coronal cerebral angiographies using intra-arterial injections were acquired with a GRE sequence with in-plane resolution of $0.27 \mathrm{~mm}$ and matrix size $256 \times 128$, and $2 \mathrm{D}$ coronal cerebral angiographies were acquired with a bSSFP sequence with in-plane resolution of $0.55 \mathrm{~mm}$ and matrix size $128 \times 64$.

The bSSFP sequence provides higher SNR in phantoms than the GRE sequence. Similarly, intravenous injections are imaged with higher SNR with the bSSFP sequence, where the signal destruction of the GRE sequence is avoided. However, for intra-arterial injections, the bSSFP sequence results in strong artefacts, and the GRE sequence is preferred.

Hyperpolarized MRA presents many challenges and cannot currently compete with conventional contrast enhanced MRA. Further research may change this since hyperpolarization is still an immature methodology. 


\section{Introduction}

Angiographic techniques are important in the diagnosis and treatment of medical conditions such as emboli, stenosis and intracranial aneurysms. The most frequently applied angiographic techniques are ultrasound imaging (US), computed tomography (CT), and nuclear medicine imaging such as PET [1]. US is non-invasive, but has its limitations in imaging of the cerebrovascular system, where cranium and tissue-bone interfaces cause attenuation and disturbance of the signal. CT provides high resolution images within short acquisition time, but the radiation exposure is a major limitation of repeated use. PET angiography can provide information about the functional metrics in the vascular system, however the low spatial resolution and the administration of radioactive contrast agent makes PET less preferable [2,3]. Magnetic resonance angiography (MRA) is generally used with a contrast agent based on e.g. gadolinium [4] that reduces the $\mathrm{T}_{1}$ of the blood, but flow-dependent techniques such as time-offlight (TOF) or phase contrast can also be employed without injections [5,6]. One class of MRA contrast agents includes blood pool agents such as Clariscan ${ }^{\mathrm{TM}}$; ultra-small super paramagnetic iron oxide particles that stay in circulation for hours [7]. Thus, the arterial vascular system alone can be imaged during the first pass of the contrast agent, whereas the entire vascular system can be acquired in a large time window allowing signal averaging for high resolution. Time-resolved fast imaging can be applied to capture the dynamics [8].

\section{Hyperpolarized ${ }^{13} \mathrm{C} M R A$}

Conventional coronary proton MRA acquisition time depends on parameters such as matrix size and repetition time, and is typically acquired over several minutes [9]. Thus, respiratory and cardiac motion, as well as movements of the patient will affect the image quality. The need for faster image acquisition has catalyzed research in hyperpolarized (HP) MRI, where the signal can be enhanced by orders of magnitude compared to the thermal signal [10]. The polarization is obtained via dissolution Dynamic Nuclear Polarization (DNP): The sample is mixed with an Electron Paramagnetic Agent (EPA) and cooled to low temperature in a magnetic field, by which the unpaired electrons of the EPA reach a polarization of unity. The polarization of the electrons is transferred to the nuclear spins by irradiating with microwaves at the resonance frequency of the electrons. The high polarization of the nuclear spins are preserved to the liquid phase by rapidly dissolving in a dissolution medium, before the agent is injected. The polarization of the injected volume is more than 4 orders of magnitude larger than the thermal signal of ${ }^{13} \mathrm{C}$. 
Therefore, the signal in a ${ }^{13} \mathrm{C}$ image obtained after injection of a hyperpolarized substance is proportional to the concentration of the agent in question, and the image acts as a qualitative map of perfusion [11-14]. Likewise, the conversion of hyperpolarized ${ }^{13} \mathrm{C}$-labelled compounds such as pyruvate can be followed with Magnetic Resonance Spectroscopic Imaging (MRSI)[15-17], and the metabolic activity monitored [18].

Hyperpolarized MRA provides imaging of the injected substance in the vascular system, and thus provides a direct signal from the hyperpolarized spins in the blood. In addition, hyperpolarized MRA is not dependent on flow velocity as e.g. TOF-imaging, where flow close to the vessel walls appears with a low signal due to low flow velocity. In 2002 Golman et al. demonstrated that angiography in rats using hyperpolarized ${ }^{13} \mathrm{C}$ is possible with bis1,1-(hydroxymethyl)-[1- $\left.{ }^{13} \mathrm{C}\right]$ cyclopropane-D8 (HP001) as imaging agent and demonstrated that both ParaHydrogenInduced Polarization (PHIP)[19] and dissolution DNP [20] can be used for hyperpolarized ${ }^{13}$ C angiography with i.v. agent administration (Figure 1). Svensson et al. [21] studied ${ }^{13} \mathrm{C}$ cerebral MRA in the rat and found that high SNR can be obtained in both the head and neck area (Figure 2) and in the thoracic/abdominal region using the balanced steady state free precision pulse sequence (bSSFP). Later, Olsson et al. [22] presented a study on hyperpolarized ${ }^{13} \mathrm{C}$ coronary MRA in a pig model with i.a. injection. Recently, hyperpolarized $\mathrm{H}_{2} \mathrm{O}$ has been applied in angiography with the advantage of applicability on clinical scanners with ${ }^{1} \mathrm{H}$ channels only, as well as higher achievable magnetization due to a larger magnetic moment [23].

For cerebral angiography in the rat, the hyperpolarized substance is typically injected using either intravenous (i.v.) injection through one of the tail veins, or intra-arterial (i.a.) injection where a catheter is operated into one of the carotid arteries. The injection method affect the system in various ways and e.g. movement of the catheter and the reaction of the vessels to increased pressure will result in difference in signal-to-noise ratio (SNR) and artefacts in the images. Thus, the MR sequence must be chosen and optimized in accordance with the injection method. Furthermore, the injection type will enhance specific characteristics of the arterial and venous network. An even distribution in the vasculature should be observed with an intravenous injection when the hyperpolarized substance is distributed through the heart of the animal. When the substance is injected in a carotid artery, only arteries branching from that specific carotid artery as well as the corresponding veins are highlighted.

\section{${ }^{13}$ C agent and MRI considerations}


The ${ }^{13} \mathrm{C}$-labelled agent HP001 is chosen for its long $\mathrm{T}_{1}$ (in vitro, $2.35 \mathrm{~T}: \mathrm{T}_{1} \approx 82 \mathrm{~s}, \mathrm{~T}_{2} \approx 18 \mathrm{~s}$; in vivo, $2.35 \mathrm{~T}: \mathrm{T}_{1} \approx$ $38 \mathrm{~s}, \mathrm{~T}_{2} \approx 1.3 \mathrm{~s}$ )[21]. This means that the signal decay should be caused mainly by dilution in the vasculature and extravasation of the agent to the surrounding tissue, and to a smaller extent by relaxation processes.

The bSSFP imaging technique has been demonstrated to be a rapid, high-SNR technique for angiography and spectroscopic applications using hyperpolarized substances (Figure 2)[21,22,24,25]. The gradient echo (GRE) technique has been used extensively for single resonance HP lung imaging [26-28], but less for HP- angiography using ${ }^{13} \mathrm{C}$-labeled substances. A theoretical basis and in vitro measurement comparison between bSSFP and GRE for the HP angiography application was performed by Svensson et al. [21], but in vivo comparisons between the combinations of the two imaging techniques (bSSFP and GRE) and the two injection techniques (i.v. and i.a.) is to our knowledge not performed.

In this review we demonstrate the current possibilities of a hyperpolarized ${ }^{13} \mathrm{C}$-labelled agent for MRA and investigate the difference between intravenous and intra-arterial injection with the GRE and bSSFP imaging sequences.

\section{Methods}

\section{$\underline{\text { Polarization }}$}

Bis-1,1-(hydroxymethyl)-[1- $\left.{ }^{13} \mathrm{C}\right]$ cyclopropane-D8 (HP001) was used as the imaging agent to obtain long relaxation times of the ${ }^{13} \mathrm{C}$ nuclei. Trityl OX063 (Oxford Instruments, Abingdon UK) was used as paramagnetic agent. A batch consisting of $18.0 \mathrm{mg}$ OX063, $500 \mathrm{mg}$ HP001, $180 \mathrm{mg}$ water and $15 \mu \mathrm{L}$ Dotarem (1:10 dilution, Guerbet, Paris, France) was prepared. $100 \mu \mathrm{L}$ sample was polarized using an Oxford Instruments Hypersense, $3.35 \mathrm{~T}, 1.4 \mathrm{~K}$, by irradiation with microwaves at $94.112 \mathrm{GHz}$ for one hour. The polarization time constant was $1800 \mathrm{~s}$. The sample was dissolved in $1 \mathrm{~mL}$ PBS buffer with $0.01 \mathrm{~g} / \mathrm{L}$ EDTA diluted in $3 \mathrm{~mL} \mathrm{NaCl}(9 \mathrm{~g} / \mathrm{L})$. The ${ }^{13} \mathrm{C}$ concentration in the final solution was $100 \mathrm{mmol} / \mathrm{L}$. The solution temperature was approximately $37{ }^{\circ} \mathrm{C}$ and the $\mathrm{pH} 7.5-8$. The time from dissolution to injection start was $14-18 \mathrm{~s}$ and the polarization was $20-25 \%$ at time of injection.

\section{$\underline{\text { Animal Preparation }}$}


All animal experiments were carried out in compliance with the guidelines for use and care of laboratory animals and were approved by the Danish Inspectorate of Animal Experiments, approval 2012-15-2934-00244.

Male Sprague Dawley rats weighing 240-320 g were anaesthetized with $1.8 \%$ isoflurane in air mixed with $5 \%$ oxygen adjusted to a respiration rate of $75 \pm 10 \mathrm{~min}^{-1}$ and mounted in an animal holder. The body temperature was kept at $38 \pm 1{ }^{\circ} \mathrm{C}$ with hot air flow.

Tail vein catherization: A catheter was inserted in a tail vein for i.v. contrast agent administration. A volume of 1.5 $\mathrm{mL}$ was injected over 6-9 $\mathrm{s}$.

Carotid artery catherization: Carotid artery injections were prepared by incision in the ventral neck of the rat and isolation of the right carotid artery, before inserting and securing the catheter in the artery. The catheter was fixed to the skin with tape. A volume of $1.0 \mathrm{~mL}$ was injected over 6-9 s.

MR scanner system and pulse sequences:

Data was acquired on a $4.7 \mathrm{~T}$ imaging system (Agilent, Direct Drive, VnmrJ 4.0) using a ${ }^{13} \mathrm{C} /{ }^{1} \mathrm{H}$ RF volume coil combined with a ${ }^{13} \mathrm{C}$ 4-channel surface array coil (receive-only, $50 \mathrm{~mm}$ inner diameter, RAPID Biomedical GmbH, Germany). The receive coil was placed dorsal with the animal supine. Figure 3 shows the profile of the coil sensitivity measured as the sum-of-squares image of a CSI acquisition of a spherical bicarbonate phantom. The position of the GRE slab is indicated as the shaded area. The bSSFP slab is overlapping, but extends $10 \mathrm{~mm}$ in the anterior direction. The sensitivity is highest in the region closest to the coil elements, thus the sensitivity of the vessels in this region is more than twice the sensitivity in the outer region of the slab. The system was shimmed to a proton line width of less than $110 \mathrm{~Hz}$ over the field-of-view (FOV). The image reconstruction applied the sum-ofsquares algorithm where the signal in each pixel is the square root of the sum of the squares of the signal from each individual array coil element. The background noise is measured in a ROI of 20x20 pixels in an area away from the hyperpolarized signal.

Coil Sensitivity measurements: The coil sensitivity is assessed with a CSI pulse sequence with FOV $80 \times 80 \mathrm{~mm}^{2}$, matrix size $16 \times 16, \alpha=10^{\circ}, \mathrm{TR}=75 \mathrm{~ms}$ on a spherical phantom of $1.0 \mathrm{M}$ bicarbonate ( $38 \mathrm{~mm}$ diameter). 
$\underline{H P-}{ }^{13} C-M R A-b S S F P:$ The hyperpolarized signals were acquired using a single-shot bSSFP sequence with $110^{\circ}$ flip angle $(\alpha)$. An $\alpha / 2$-pulse preparation pulse was implemented at time TR/2 before the first excitation pulse to approach a steady state like situation faster and a corresponding flip-back pulse was implemented at the end of each image acquisition to restore the longitudinal magnetization for the following time frame. The angiographic images had a Field-Of-View (FOV) of 70x35 $\mathrm{mm}^{2}$ and a slab thickness of $30 \mathrm{~mm}$ placed coronal in the head and neck region. The matrix size was $128 \times 64$. The repetition time and echo time were set to minimum, TR/TE $=6.95 / 3.48 \mathrm{~ms}$. A bandwidth of $50 \mathrm{kHz}$ yielded a total image acquisition of $450 \mathrm{~ms}$ for each image. The sequence was started when the solution had been fully injected to capture the maximum possible signal.

$\underline{H P-}{ }^{13} C-M R A, G R E:$ The GRE sequence was applied with an FOV of 70x35 $\mathrm{mm}^{2}$, a slab thickness of $20 \mathrm{~mm}$ and with matrix size $256 \times 128$. The pulse length was $2 \mathrm{~ms}$ and the repetition and echo times were TR/TE $=10.16 / 5.10$ ms. The total acquisition time for an image was $1300 \mathrm{~ms}$ with $\alpha=5^{\circ}$ and imaging was repeated over 30 time frames. Acquisition was started when injection was initiated to capture the maximum signal strength.

${ }^{1} H M R A$ : Proton angiographic images were acquired with a $1 \mathrm{H}$ quadrature coil (M2M imaging corporation, Cleveland, Ohio, USA) with inner diameter of $35 \mathrm{~mm}$. A 3D GRE sequence with TR/TE $=90 / 2.47 \mathrm{~ms}, \alpha=40^{\circ}$, FOV of $30 \times 35 \times 20 \mathrm{~mm}^{3}$, matrix size $256 \times 256 \times 64$ was used after an intravenous injection of $50 \mu \mathrm{L}$ Clariscan ${ }^{\mathrm{TM}}$ (NC100150, NYCOMED IMAGING AS, Oslo, Norway). The total acquisition time was 25 minutes.

\section{Results}

${ }^{1} H M R A$ : Figure 4 shows a contrast enhanced ${ }^{1} \mathrm{H}$ 3D GRE image of the cerebral vascular system. The large arrow indicates the superior sagittal sinus (SSS), the small arrows indicate the transverse sinuses (TRS) and the dotted arrows indicate the superficial temporal vein (STEMV), posterior facial.

$\underline{H P-}{ }^{13} C-M R A, G R E:$ An excitation pulse flip angle of $5^{\circ}$ was found the optimal compromise between signal strength and conversion of magnetization during the time series in a separate study (data not shown). A time series of 8 frames during intra-arterial injection is shown in Figure 5 . The best image is the $4^{\text {th }}$ time frame. The rat brain was centered in the FOV, and arteries and veins of the right side of the head appear with the largest denseness around the brain and towards the nose region. Only a few veins appear in the left half of the image, and only minor signal contribution is visible in the eye region of the right side. Figure 6 shows the $4^{\text {th }}$ frame as overlay on the proton 
image. Though the two angiographies are obtained on different animals and positioning, the images are in agreement and major venal structures (SSS, TRS, STEMV) of the right side are identified.

A series of 3 consecutive intra-arterial injections of hyperpolarized HP001 in an animal with intervals of approximately one hour gave SNR 26, 19 and 20, as measured as the ratio between the largest signal observed and the background noise, and demonstrates reproducibility of the result.

18 injections in 10 animals yielded SNR of 17.6 \pm 4.5 (Mean \pm SD).

The corresponding GRE acquisition after an intravenous injection yielded signal from the larger veins but with SNR lower than 10 (acquired in 3 animals, image not shown).

$\underline{H P-}{ }^{13} C-M R A, b S S F P$ : The signal acquisition bandwidth was studied in the range of $20-100 \mathrm{kHz}$, and we found 50 $\mathrm{kHz}$ to be the optimal balance between high signal and minimal flow artefacts. The sequence was started when the solution had been fully injected to capture the maximum possible signal. In separate experiments (data not shown), the flip angle for the bSSFP- sequence was optimized on thermal phantom experiments where a flip angle of $110^{\circ}$ yielded a $22 \%$ higher SNR than the corresponding experiment with $60^{\circ}$ flip angle and where flip angles higher than $110^{\circ}$ did experiments on HP in vivo experiments with the bSSFP (data not shown). A time series of bSSFP acquired images with one second intervals is shown in Figure 7. SNR of 36 is observed in the first frame and the signal decays rapidly in the following frames. The larger veins (SSS, TRS, STEMV) identified from the overlay appear distinctly in a symmetrical shape, and smaller venal structure appears towards the nose region.

5 injections in 4 different animals yielded SNR of 31.3 \pm 20.0 (Mean \pm SD).

\section{Discussion}

In this study we injected a dose close to the anticipated clinical dose of ca. $0.4 \mathrm{mmol} / \mathrm{kg}$, which is only $25-50 \%$ of the dose used in previous studies with a similar polarization. We obtained results similar to Golman et al., where an SNR of 34 was demonstrated in a $128 \times 128$ matrix $\left(70 \times 70 \mathrm{~mm}^{2}\right)$ in the rat heart with an injection of 2-3 mL of 200 $\mathrm{mM}$ (ca. $1.5 \mathrm{mmol} / \mathrm{kg}$ ) solution with polarization of ca. 15\% [19]. Svensson et al. obtained SNR of 74-76 in a lower resolution of a $64 \times 64$ matrix $\left(70 \times 70 \mathrm{~mm}^{2}\right)$ placed in the rat head and neck region with injection of 3-3.5 mL of 200 $\mathrm{mM}$ solution with a polarization of ca. $15 \%$ [21]. 
The bSSFP sequence proved best for injections through the tail vein where the physiological and physical reactions of the animal are negligible, in contrary to injections through a carotid artery, which caused minor mechanical movements of the neck and head region where the catheter is inserted, as well as possible physiological reactions in the imaged region due to the injected bolus' impact on the vessels due to pressure, $\mathrm{pH}$, temperature and tonicity. These movements caused motion artefacts in the bSSFP acquisition, whereas a GRE sequence was more robust to displacements and hence better suitable. Images of carotid artery injection acquired with bSSFP (Figure 8) showed a very high SNR of 137. Even though this large signal from the high agent concentration in the arteries was observed, the details of the vascular system were indistinguishable. The sequence was applied with TR/TE $=6.95 / 3.48 \mathrm{~ms}, \alpha=$ $60^{\circ}$ in a $128 \times 128$ matrix with FOV $60 \times 60 \mathrm{~mm}^{2}$ in a $30 \mathrm{~mm}$ slab. The strong artefacts may be caused by motion due to the injection as well as $B_{0}$-field disturbances caused by the large difference in oxygenated blood and the water introduced with the injection [29].

The contrast agent is further diluted for tail vein injections. The combination of small flip angle GRE acquisition and large dilution in the vascular system resulted in SNR below 10. Therefore, a bSSFP sequence with high flip angle is preferred.

The bSSFP-sequence had a slab thickness of $30 \mathrm{~mm}$. In some experiments, the transverse sinuses align orthogonally to the slice and thus contribute with a large signal in the projection (arrow in Figure 7). These outliers cause the large variance where single pixels have yielded signal strength much larger than the rest of the vasculature.

Large parts of the image characteristics in Figure 5 are observed throughout the series, while some features are vanishing in the later time steps. The fact that the operated carotid has been sealed off beneath the catheter explains that some of the branches have closed off during several hours of occlusion in the scanner. The image series and the consistency in the SNR indicate that polarization, injection and data acquisition is reproducible.

When the bSSFP sequence was applied following an intra-arterial injection we observe motion artefacts in the phase encoding direction as shown in Figure 8.

Polarizers for DNP have now been installed in several clinics around the world, and studies of HP ${ }^{13} \mathrm{C}$ in humans initiated [30]. As this equipment is being implemented, the application of hyperpolarized agents for angiography in will be further studied, and a potential translation towards application in clinical practice explored. 
In conclusion, intravenous and intra-arterial injection techniques were used in combinations with optimized GREand bSSFP-imaging, respectively, for rat cerebral MRA with a hyperpolarized ${ }^{13} \mathrm{C}$-labelled agent, and resulted in bSSFP-imaging being preferable for intravenous injections, and GRE-imaging being preferable for the intra-arterial injection technique.

Hyperpolarized ${ }^{13} \mathrm{C}$ MRA is currently unable to compete with contrast enhanced MRA. Considering the complexity of the method (primarily the need for polarizer hardware and the short life time of the signal) it is unlikely that it will become an attractive method. However, hyperpolarization is still an immature methodology, and there are areas where current MRA falls short. With further research into hyperpolarization to increase the available signal, and possibly increase the life time, there are opportunities for significant improvement.

\section{Conflict of interest}

The authors confirm that this article content has no conflicts of interest.

\section{Acknowledgments}

The authors want to thank Sascha Gude for her assistance with the animal preparations.

We acknowledge funding from the Danish Research Council grant 12-127232. 


\section{References}

[1] MacDonald ME, Frayne R. Cerebrovascular MRI: a review of state-of-the-art approaches, methods and techniques. NMR Biomed 2015; 28(7): 767-791.

[2] Schroeder MA, Clarke K, Neubauer S, Tyler DJ. Hyperpolarized magnetic resonance: A novel technique for the in vivo assessment of cardiovascular disease. Circulation 2011; 124: 1580-1594.

[3] O'Leary DH, Bots ML. Imaging of atherosclerosis: carotid intima-media thickness. Eur heart J 2010; 31(14): 1682-1689.

[4] Kinner S, Maderwald S, Parohl N, et al. Contrast-enhanced magnetic resonance angiography in rabbits: evaluation of the gadolinium-based agent P846 and the iron-based blood pool agent P904 in comparison with gadoterate meglumine. Invest Radiol 2011; 46: 524-529.

[5] Beckmann N. High resolution magnetic resonance angiography non-invasively reveals mouse strain differences in the cerebrovascular anatomy in vivo. Magn Reson Med 2000; 44: 252-258.

[6] Reese T, Bochelen D, Sauter A, Beckmann N, Rudin M. Magnetic resonance angiography of the rat cerebrovascular system without the use of contrast agents. NMR Biomed 1999; 12: 189-196.

[7] Howles GP, Ghaghada KB, Qi Y, Mukundan S, Johnson GA. High-resolution magnetic resonance angiography in the mouse using a nanoparticle blood-pool contrast agent. Magn Reson Med 2009; 62: $1447-1456$.

[8] Riederer SJ, Haider CR, Borisch EA, Weavers PT, Young PM. Recent advances in 3D time-resolved contrast-enhanced MR angiography. J Magn Reson Imaging 2015; 42: 3-22.

[9] Kim WY, Danias PG, Stuber M, et al. Coronary magnetic resonance angiography for the detection of coronary stenoses. N Engl J Med 2001; 345: 1863-1869.

[10] Ardenkjær-Larsen JH, Fridlund B, Gram A, et al. Increase in signal-to-noise ratio of >10,000 times in liquid-state NMR. Proc Natl Acad Sci USA 2003; 100: 10158-10163. 
[11] Grant AK, Vinogradov E, Wang X, Lenkinski RE, Alsop DC. Perfusion imaging with a freely diffusible hyperpolarized contrast agent. Magn Reson Med 2011; 66: 746-755.

[12] Johansson E, Månsson S, Wirestam R, et al. Cerebral perfusion assessment by bolus tracking using hyperpolarized ${ }^{13}$ C. Magn Reson Med 2004; 51: 464-472.

[13] Johansson E, Olsson LE, Månsson S, et al. Perfusion assessment with bolus differentiation: A technique applicable to hyperpolarized tracers. Magn Reson Med 2004; 52: 1043-1051.

[14] Lau AZ, Miller JJ, Robson MD, Tyler DJ. Cardiac perfusion imaging using hyperpolarized ${ }^{13} \mathrm{C}$ urea using flow sensitizing gradients. Magn Reson Med 2015, May 20. [Epub ahead of print].

[15] Day SE, Kettunen MI, Gallagher FA, et al. Detecting tumor response to treatment using hyperpolarized ${ }^{13} \mathrm{C}$ magnetic resonance imaging and spectroscopy. Nat Med 2007; 13: 1382-1387.

[16] Albers MJ, Bok R, Chen AP, et al. Hyperpolarized ${ }^{13} \mathrm{C}$ lactate, pyruvate, and alanine: Noninvasive biomarkers for prostate cancer detection and grading. Cancer Res 2008; 68(20): 8607-8615.

[17]Lauritzen MH, Søgaard LV, Madsen PL, Ardenkjær-Larsen JH. Hyperpolarized metabolic MR in the study of cardiac function and disease. Curr Pharm Des 2014; 20: 6162-6170(9).

[18] Dodd MS, Ball V, Bray R, et al. In vivo mouse cardiac hyperpolarized magnetic resonance spectroscopy. J Cardiovasc Magn Reson 2013; 15: 19.

[19] Golman K, Ardenkjær-Larsen JH, Svensson J, et al. ${ }^{13}$ C-angiography. Acad Radiol 2002; 9(suppl 2): 507510.

[20] Golman K, Axelsson O, Jóhannesson H, Månsson S, Olofsson C, Petersson. Parahydrogen-induced polarization in imaging: Subsecond ${ }^{13} \mathrm{C}$ angiography. Magn Reson Med 2001; 46: 1-5.

[21] Svensson J, Månsson S, Johansson E, Petersson JS, Olsson LE. Hyperpolarized ${ }^{13} \mathrm{C}$ MR angiography using trueFISP. Magn Reson Med 2003; 50: 256-262.

[22] Olsson LE, Chai C-M, Axelsson O, Karlsson M, Golman K, Petersson JS. MR coronary angiography in pigs with intraarterial injections of a hyperpolarized ${ }^{13} \mathrm{C}$ substance. Magn Reson Med 2006; 55: 731-737. 
[23] Ardenkjær-Larsen JH, Laustsen C, Bowen S, Rizi R. Hyperpolarized $\mathrm{H}_{2} \mathrm{O}$ MR angiography. Magn Reson Med 2014; 71: 50-56.

[24] Leupold J, Månsson S, Petersson JS, Hennig J, Wieben O. Fast multiecho balanced SSFP metabolite mapping of ${ }^{1} \mathrm{H}$ and hyperpolarized ${ }^{13} \mathrm{C}$ compounds. Magn Reson Mater Phy 2009; 22: 251-256.

[25] Leupold J, Wieben O, Månsson S. Fast chemical shift mapping with multiecho balanced SSFP. Magn Reson Mater Phy 2006; 19: 267-273.

[26] Kauczor HU. Hyperpolarized helium-3 gas magnetic resonance imaging of the lung. Top Magn Reson Imaging 2003; 14: 223-230.

[27] Deninger AJ, Månsson S, Petersson JS. Quantitative measurement of regional lung ventilation using ${ }^{3} \mathrm{He}$ MRI. Magn Reson Med 2002; 48: 223-232.

[28] Mugler JP, Altes TA. Hyperpolarized ${ }^{129}$ Xe MRI of the human lung. J Magn Reson Imaging 2013; 37: $313-331$.

[29] Jain V, Abdulmalik O, Propert KJ, Wehrli FW. Investigating the magnetic susceptibility properties of fresh human blood for noninvasive oxygen saturation quantification. Magn Reson Med 2012; 68: 863-867.

[30] Nelson SJ, Kurhanewicz J, Vigneron DB, et al. Metabolic imaging of patients with prostate cancer using hyperpolarized $\left[1{ }^{13} \mathrm{C}\right]$ pyruvate. Sci Transl Med 2013; 5(198): 198. ra108. 


\section{Figure legends}

Figure 1: Angiographic image of the rat heart region after i.v. injection in the tail vein. Acquired with a gradient spoiled, gradient echo sequence $\left(\mathrm{TE} / \mathrm{TR}=4 \mathrm{~ms} / 9.5 \mathrm{~ms}, \mathrm{FA}=10^{\circ}, \mathrm{FOV}=70 \times 70 \mathrm{~mm}^{2}\right.$, matrix $=128 \times 128$, slice $=30$ $\mathrm{mm}$, scan time $=2 \mathrm{~s}$ ). With permission from [19] and Elsevier.

Figure 2: First image in a series of angiograms covering the head and neck region. The image has been acquired on a $2.35 \mathrm{~T}$ animal MRI system with a trueFISP sequence $\left(\mathrm{TE} / \mathrm{TR}=1.8 \mathrm{~ms} / 3.6 \mathrm{~ms}, \mathrm{FOV}=70 \times 70 \mathrm{~mm}^{2}\right.$, and matrix $=$ 64x64, with read oversampling). Major vessels appear with SNR of 74-76, but some enhancement of other tissue is observed as well. With permission from [21] and John Wiley and Sons.

Figure 3: The vertical sensitivity profile of the coil from a sum of squares CSI image of a spherical bicarbonate phantom (small insert). The FOV and slab position of the i.v. (bSSFP) acquisitions and the thinner slab of the i.a. (GRE) acquisitions are indicated.

Figure 4: Coronal image of cerebral vessel structure acquired with a 3D gradient echo sequence after injection of NC100150. The large arrow indicates the superior sagittal sinus, the small arrows indicate the transverse sinuses, and the dotted arrows indicate the superficial temporal vein.

Figure 5: Time series of GRE images initiated after injection of hyperpolarized HP001 through the right carotid artery. $1.3 \mathrm{~s}$ between each frame.

Figure 6: Image of GRE sequence ( $4^{\text {th }}$ frame) initiated after injection of hyperpolarized HP001 through the right carotid artery as overlay on the proton image of Figure 4. The veins SSS, TRS (right) and STEMV (right) coincide and the structure of finer vessels appear in the overlay.

Figure 7: bSSFP time series after finished injection of hyperpolarized HP001 though the tail vein, $1 \mathrm{~s}$ between frames. Right image shows the first image as overlay on the proton image of Figure 4. The veins SSS, TRS and STEMV coincide while smaller vessels are not distinguishable.

Figure 8: bSSFP acquired image after intra-arterial injection demonstrates high SNR of 137 and artefacts due to motion and susceptibility change. 
Figures

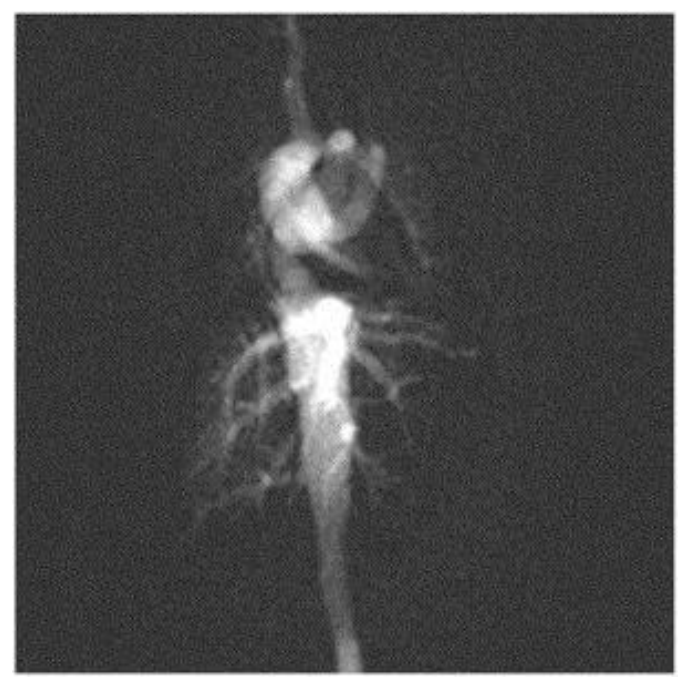

Figure 1

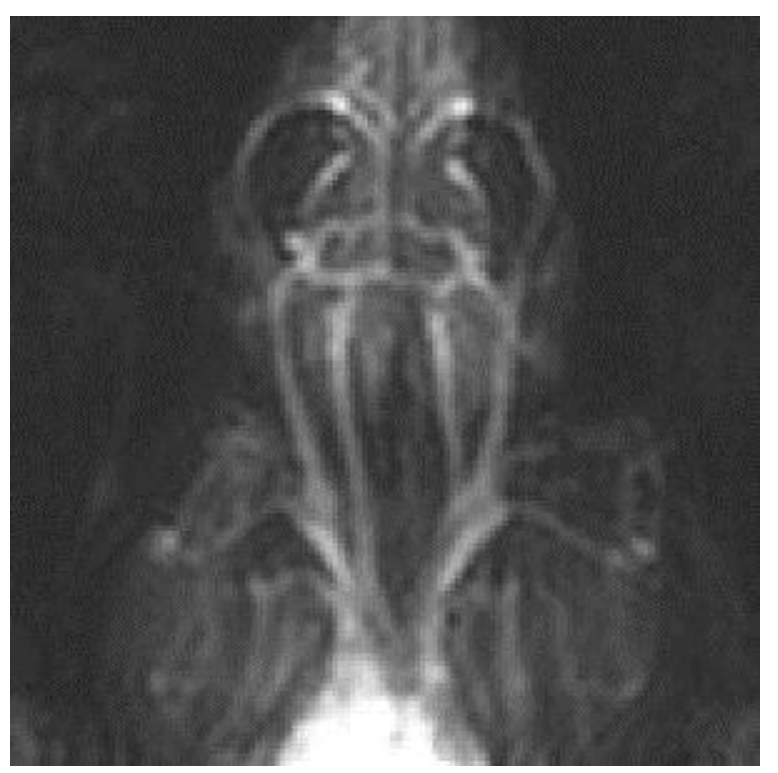

Figure 2 


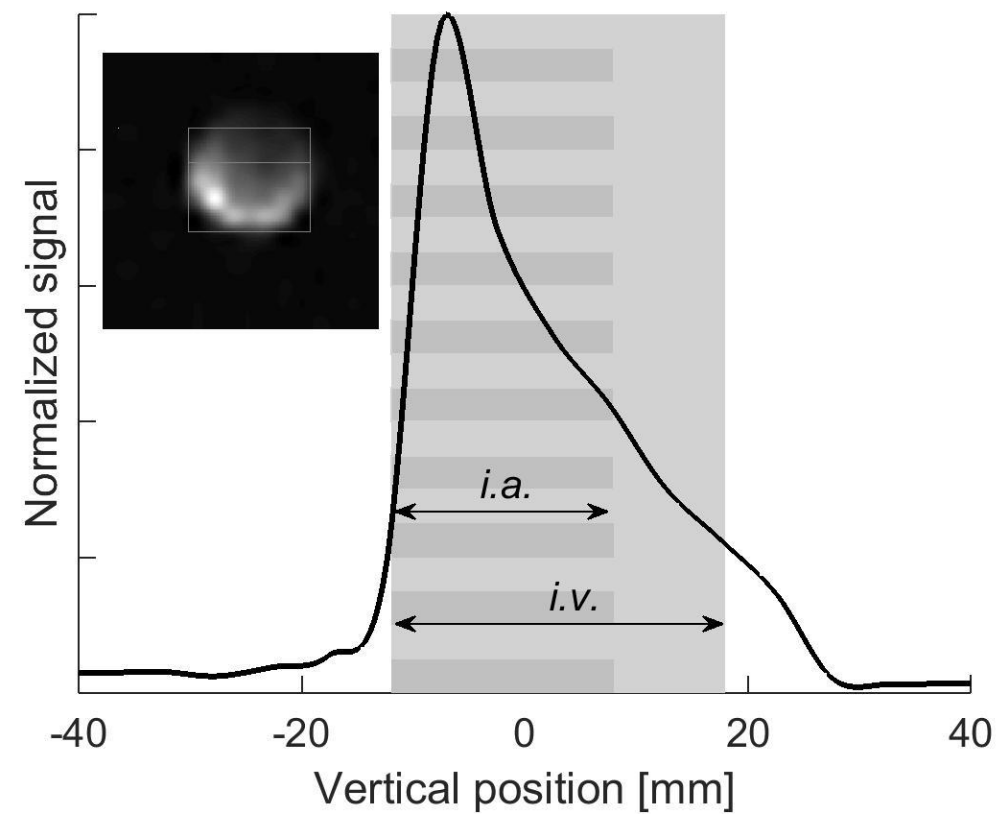

Figure 3

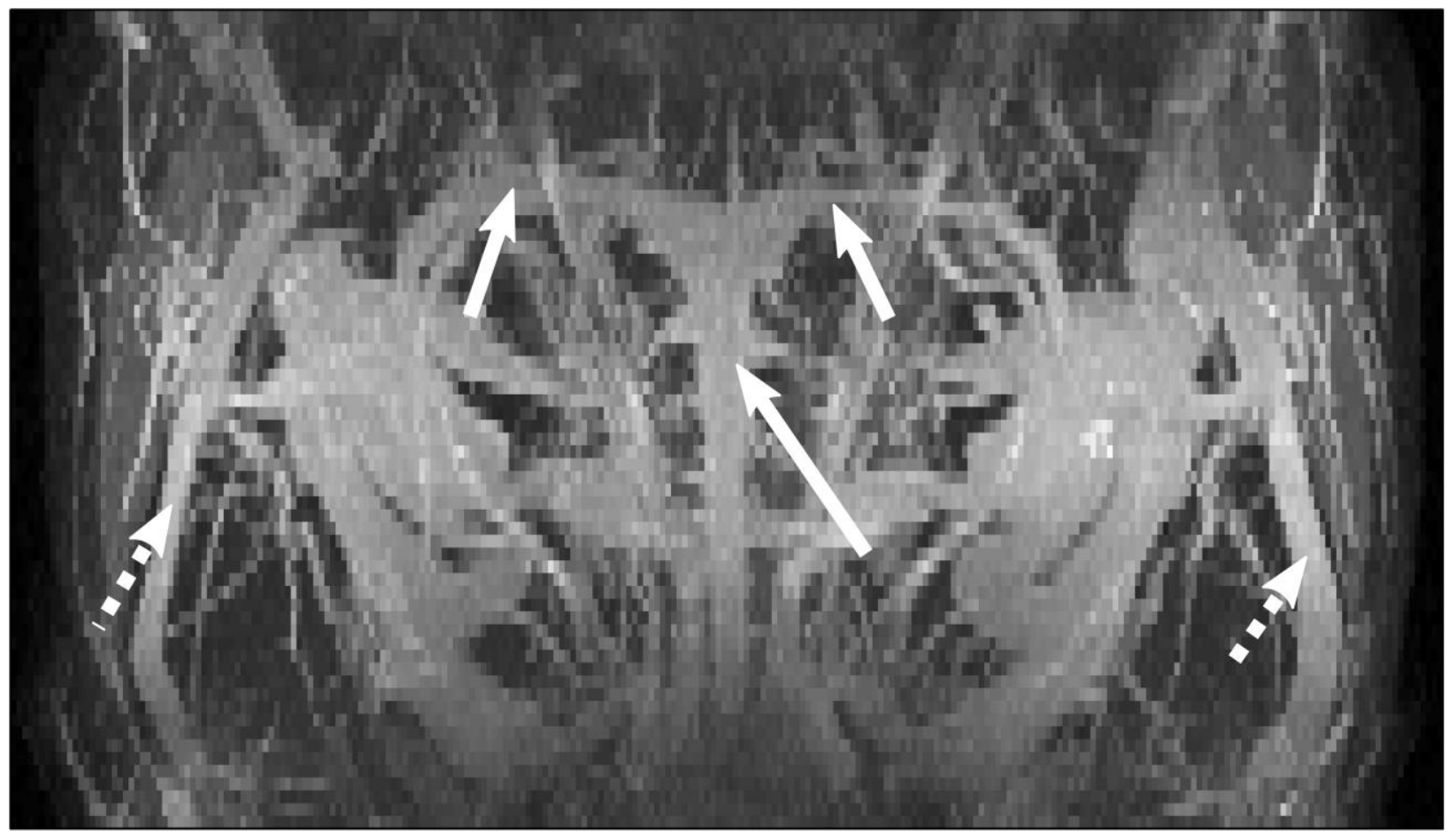

Figure 4 


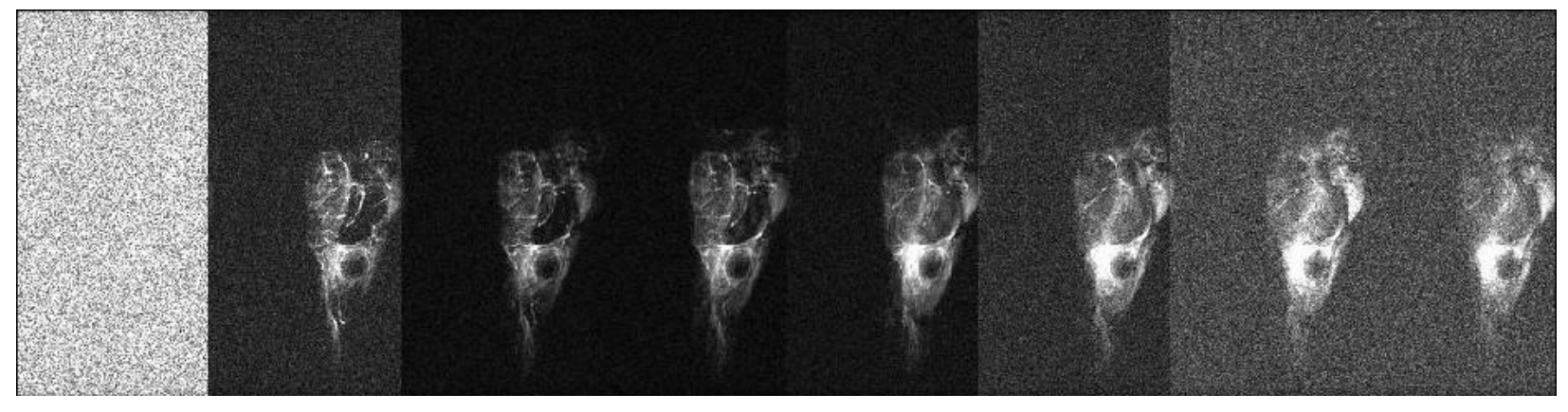

Figure 5

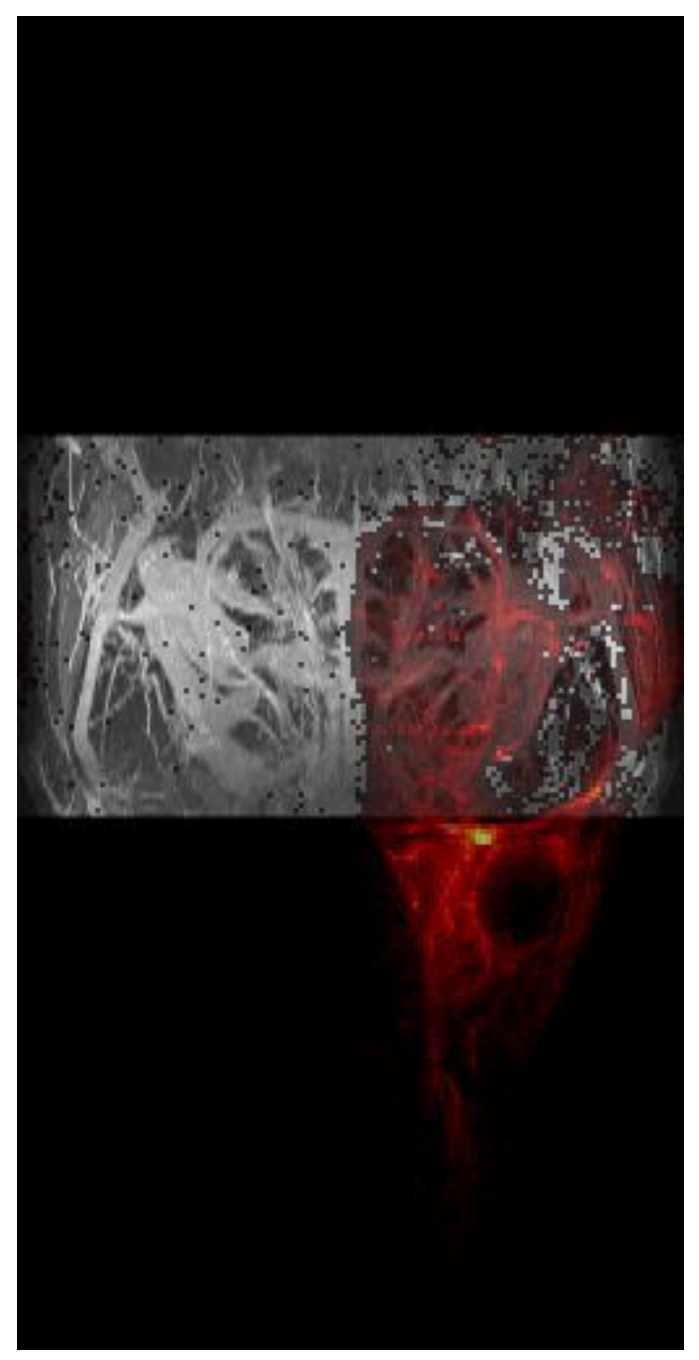

Figure 6 


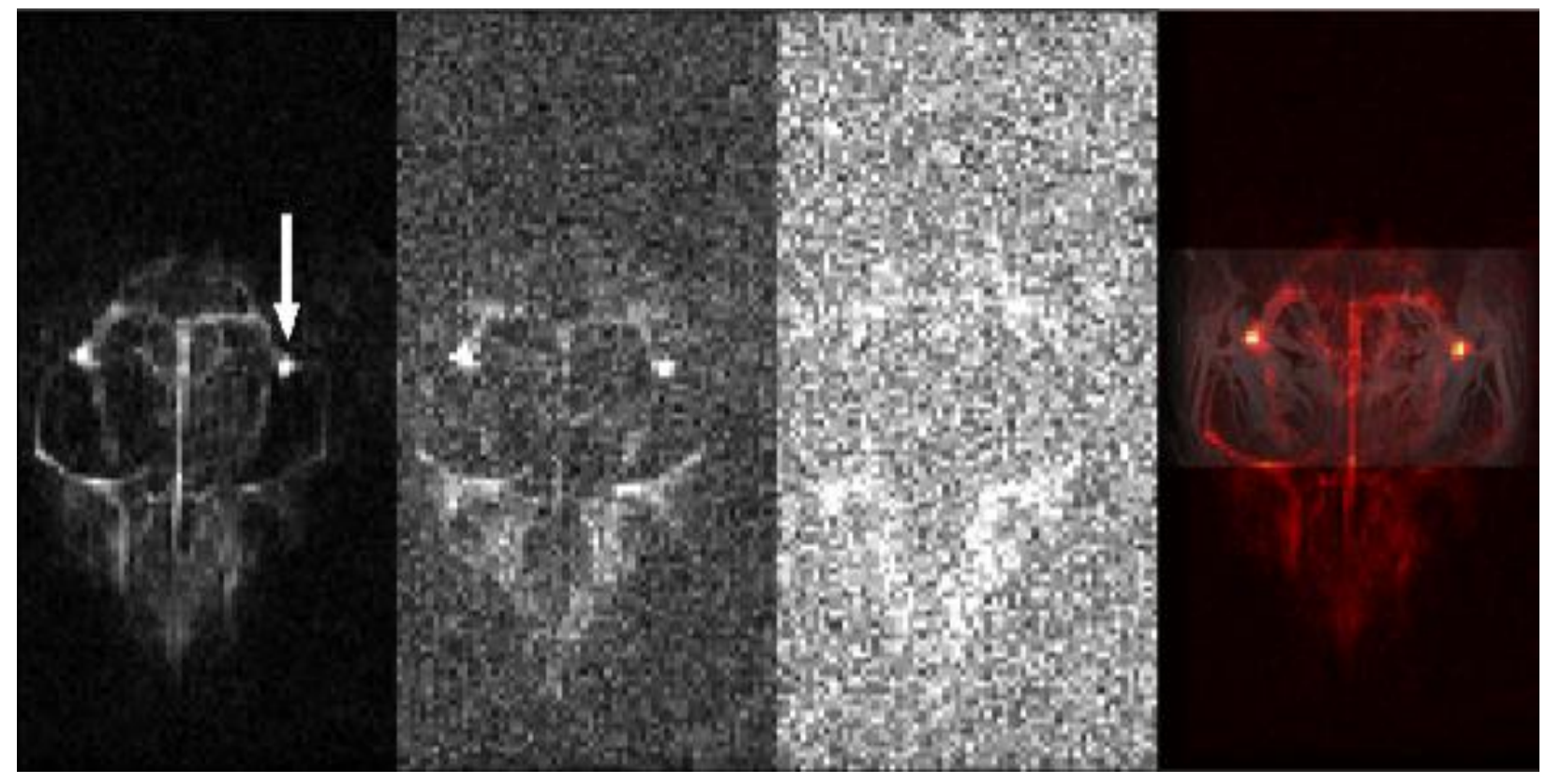

Figure 7

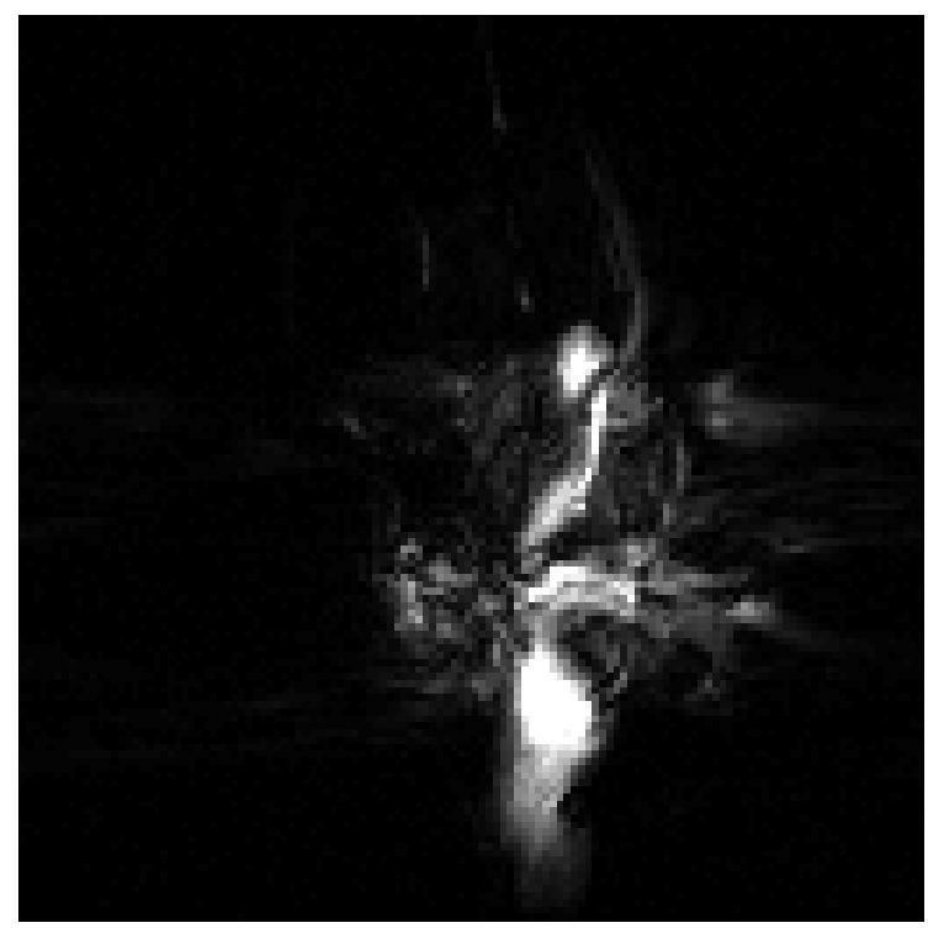

Figure 8 
